\title{
¿Quimera o Fénix? El recorrido europeo y latinoamericano hacia un derecho común de contratos*
}

\section{Alfredo Ferrante ${ }^{\star *}$}

Resumen: En el trabajo se realizan algunas reflexiones críticas sobre el proceso de unificación del derecho privado europeo que, después de un cuarto de siglo, se encuentra en un proceso menguante y vacilante. $\mathrm{Al}$ mismo tiempo se hace hincapié en las nuevas iniciativas que se han creado en Latinoamérica y en la importancia que la Convención de Viena sigue revistiendo en la actualidad en ambos continentes.

Palabras clave: Unificación del derecho civil, Normativa común de compraventa europea, Principios latinoamericanos de derecho de contractos, Convención de Viena, Principios Unidroit.

\section{Chimera or Phoenix? The European and Latin American Processes of the Unification of Contract Law}

Aвstract: This paper gives some critical perspectives on the Process of the Unification of European Private Law, which after a quarter century, is diminish-

Fecha de recepción: $\mathrm{I}_{3}$ de octubre de 2015. Fecha de aceptación: 2 I de marzo de 2016.

Para citar el artículo: A. Ferrante, “¿Quimera o Fénix? El recorrido europeo y latinoamericano hacia un derecho común de contratos", Revista de Derecho Privado, Universidad Externado de Colombia, n. ${ }^{\circ}$ 30, enero-junio de 20I6, I07-I27. DOI: http://dx.doi.org/Io.1860I/or 234366.n30.05

* Doctor en Derecho con mención europea por la Universidad de Oviedo, Oviedo, España. Profesor de la Universidad Alberto Hurtado, Santiago de Chile, Chile. Miembro del European Law Institute. Contacto: aferrante@uahurtado.cl 
ing and faltering. This paper also emphasizes the newly created initiatives in Latin America and the current importance, in both continents, of the Vienna Convention.

Keywords: Unification of Private Law, Common European Sales Law, Latin American Principles of Contract Law, cisg, Vienna Convention, Unidroit Principles.

Sumario. i. El Código Europeo Común de Derecho Privado y la profecía diabólica. II. ¿C-ISG O CI-SG? O ¿CESL O CL-ES? III. La tendencia de los proyectos de unificación normativa hacia la internacionalización privatista. A. Los Principios Unidroit. B. La unificación del derecho latinoamericano y la Convención de Viena. Iv. La nueva apuesta en Latinoamérica sobre derechos de contratos.

\section{El Código Europeo común de Derecho Privado y la profecía diabólica}

Aunque el germen doctrinal de la integración jurídica europea se ubica en el año ${ }_{197} 6^{[\mathrm{r}]}$ y empieza gracias a un trato de favor ${ }^{2}$ en el año ${ }_{19} 82^{[3]}$, el verdadero proceso para un "Código Europeo común de Derecho Privado" empieza en r989 ${ }^{[4]}$.

El apoyo $^{5}$ a la Comisión Lando, la creación de una network especializada en derecho privado europeo (Foint Network on European Private Law), la Red de Expertos Interesados (Network of Stakebolder Experts) ${ }^{6}$, el trabajo del Study Group (Study Group on a European Civil Code), del Acquis Group (Research Group on Exis-

I La idea estuvo dirigida desde i976 por Ole LANDo, quien buscó miembros para la que será la "Commission pour le droit européen du contrat" (Commission on European Contract Law, CECL): ver Lando, O., "My life as a lawyer", Zeitschrift für Europäisches Privatrecht, n. ${ }^{\circ}$ 3, 2002, 5 I 9.

2 La Commission on European Contract Law recibe algunas ayudas europeas gracias a la intermediación del Director General del Servicio Legal de la Comisión Europa, Claus Ehlermann, amigo de Ole Lando, y las ayudas económicas a la Comisión terminan justamente en I995, cuando Ehlermann deja el servicio legal; desde esta fecha las ayudas proceden esencialmente de instituciones alemanas: ver Lando, "My life as a lawyer", cit., 517, 5 I 9 y 520.

3 Cfr. ibíd., 520. Sobre la Comisión, ver Lando, O., "European Contract Law", The American Journal of Contract Law, I983, 653 ss., en particular 655 ss.; Riedel, K., "The Work of the Lando-Commission from an Alternative Viewpoint", European Review of Private Law, 200o, v. 8, n. ${ }^{\circ}$ I, 79 ss.

4 Resolución del Parlamento Europeo del 26 de mayo de i 989 sobre un esfuerzo para armonizar el derecho privado de los Estados miembros (A2-I 57/89), DOCE, C I 58 del 26 de junio de I989, 400 y 401 .

5 Resolución del Parlamento Europeo del 6 de mayo de i 994 sobre la armonización de determinados sectores del derecho privado de los Estados miembros (A3-0329/94), DOCE, C 205, del 25 de julio de 1994,5 I8-519. El apoyo económico termina en I995: ver supra nota 2.

6 Para más detalles ver: http://www.copecl.org/ 
ting EC Private Law)7 , de la Association Henri Capitant des Amis de la Culture Furidique Française y de la Société de Legislation Comparée 8 y su esfuerzo de traducirlo9, han quedado prácticamente en agua de borrajas.

El $\operatorname{DCFR}^{\mathrm{IO}}$, hijo de los PECL ${ }^{\mathrm{II}}$, ya reducido en la $\mathrm{CESL}^{\mathrm{I} 2}$, pasa, de ser considerado un instrumento de derecho indicativo o de soft law ${ }^{13}$, o "simplemente un

7 von Bar, C.; Clive, E. y Schulte Nölke, H. (eds.), Principles, Definitions and Model Rules of European Private Law. Draft Common Frame of Reference (DCFR), Outline edition, Munich, Sellier, 2009. La versión retoca la anterior de 2008 y su elaboración se debe al Study Group (Study Group on a European Civil Code) y al Acquis Group (Research Group on Existing EC Private Law). El Proyecto fue financiado por el vi Programa marco de investigación (2002-2006), Decisión n. ${ }^{\circ}$ I 5 I 3/2002/CE del Parlamento Europeo y del Consejo, del 27 de junio de 2002, relativa al sexto Programa marco de la Comunidad Europea para acciones de investigación, desarrollo tecnológico y demostración, destinado a contribuir a la creación del Espacio Europeo de Investigación y a la innovación (2002-2006) DOCE L 232 del 29 de agosto 2002, I-33. A lo largo de todo el procedimiento se ha creado una Red Conjunta en Derecho Privado Europeo (Foint Network on European Private Law) y la Red de Expertos Interesados (Network of Stakeholder Experts): para más detalles ver: http://www.copecl.org/.

8 Aa.vv., Terminologie contractuelle commune - Projet de cadre commun de référence, SocIÉTÉ DE LÉgislation Comparée et Association Henri Capitant (ed.), coll. "Droit privé comparé et européen”, vol. 6, Munich, Sellier, 2008; AA.vv., Principes contractuels communes - Projet de cadre commun de référence, Société de Législation Comparée et Association Henri Capitant (ed.), coll. "Droit privé comparé et européen”, v. 7, Munich, Sellier, 2008.

9 AA.vv., European Contract Law. Materials for a Common Frame of Reference: Terminology. Guiding Principle, Model Rules, Société de Législation Comparée et Association Henri Capitant (ed.) Bénédicte Fauvarque-Cosson et Denis Mazeaud (coord.), Munich, Sellier, 20o8. Debe destacarse aquí que la edición ha contado con el soporte de la Fondation pour el droit continental.

Io A la interim outline edition de 2008 siguen las outline y full edition de 2009: STUdy Group on a European Civil Code y Research Group on the Existing EC Private Law (Acquis Group), Principles, Definition and Model Rules of European Private Law. Draft Common Frame of Reference (DCFR). Full Edition, iII, edited by von Bar, Clive, Schulze, Munich, Sellier, 2009.

I I El proyecto dura I 7 años y necesita de 22 encuentros antes de estrenarse: LANDo, "My life as a lawyer", cit., 520 .

La versión en inglés es de I995, la francesa de I997, la segunda edición es de 2000, a las que se añade una Parte in en 2003. Lando, O. y Beale, H., Principles of European Contract Law, prepared by the Commission on European Contract Law (Ole Lando, Hugh Beale [eds.]), Nijhoff, Dordrecht 1995 (Les Principes du droit européen du contrat: l'exécution, l'inexécution et ses suites, Isabelle de Lamberterie, Georges Rouhette, Denis Tallon [trad.], Documentation française, Paris, I997); Lando, O. y Beale, H., Principles of European Contract Law. Parts I and II, combined and revised for the Commission on European Contract Law (Ole Lando, Hugh Beale, eds.), Kluwer Law International, The Hague, London, Boston, 2000. Lando, O.; Clive, E.; Prüm, A. y Zimmermann, R., Principles of European Contrat Law. Part III, por la Commission on European Contract Law (Ole Lando, Eric Clive, Rene Prüm, Reinard Zimmermann [eds.]), Kluwer Law International, Hague, London, Boston, 2003. Por la versión completa en francés: Lando, O. y Beale, H., Principles of European Contract Law. Commission pour le droit européen du contrat, version française préparée par Georges Rouhette, avec le concours de Isabelle de Lamberterie, Denis Tallon, Claude Witz, Société de législation comparée, Paris, 2003.

I 2 Propuesta de Reglamento del Parlamento Europeo y del Consejo relativo a una Normativa común de compraventa europea [сом(20I I) 635 final] del I I de octubre de 20 I I.

I 3 En este sentido, ver Resolución del Parlamento Europeo, del I 2 de diciembre de 2007, sobre el derecho contractual europeo, Texto aprobado P6_TA(2007)o6 I 5. 
documento académico" ${ }^{\mathrm{I} 4}$, a aspirar a convertirse en una realidad jurídica ${ }^{\mathrm{I} 5}$, para luego condenársele a ser definitivamente coartado. Este proceso ha pasado a ser algo totalmente diferente de lo que se quería lograr en un comienzo.

Después de poco más de 25 años lo que ha quedado, desde febrero de 2014, ha sido un reglamento sobre un instrumento que no sólo es optativo ${ }^{16}$, sino que está reducido a un limitado ámbito del derecho civil. La limitación se aprecia en una doble vertiente: a) limitado a compraventa de bienes, de suministro de contenidos digitales y de prestación de servicios, y b) exclusivamente en su modalidad "a distancia y sobre todo en línea" ${ }^{7}$.

Lo que era un proyecto de unificación del derecho privado muta, en su último coletazo, en un instrumento que no solamente huye del más amplio concepto de derecho privado, sino que se convierte en una herramienta de derecho internacional, referida a relaciones jurídicas transfronterizas.

El mismo Parlamento, con su Resolución de febrero de 2014, se contradice y huye de su originaria postura que, por un lado, "lamenta(ba) que la Comisión ${ }^{18}$ haya limitado sorprendentemente su comunicación al Derecho contrac-

I4 En estos términos la Resolución del Parlamento Europeo, del 3 de septiembre de 2008, sobre un Marco Común de Referencia para el Derecho contractual europeo, P6_TA(2008)0397. P. ej., ver Fauvarque-Cosson, B., "Vers un droit commun européen de la vente", Recueil Dalloz, 20 2 2, 34.

I 5 El I 7 de septiembre de 20I3, en la página web oficial del Parlamento Europeo, se informó que el Comité de Asuntos Jurídicos apoyaría la propuesta de reglamento sobre la compraventa; ver: www.europarl.europa.eu/news/en/news-room/content/201 309 I 6IPR 2002 5/html/CommonEuropean-Sales-Law-backed-by-legal-affairs-MEPS, REF.: 20I309I6IPR2002, consultada el 24 de octubre de 2014.

I6 Sobre la naturaleza jurídica del nuevo instrumento y las siete posibilidades originariamente planteadas, ver Libro verde sobre opciones para avanzar hacia un Derecho contractual europeo para consumidores y empresas, del I de julio de 2010, сом(2010) 348 final.

I 7 Es esta la enmienda que el Parlamento Europeo introduce el pasado febrero al texto de la Enmienda n. ${ }^{\circ} 26$, por una resolución que de momento es provisional, por la que se modifica el artículo I del Anexo CESL: "Estas normas pueden utilizarse para regular las transacciones transfronterizas de compraventa de bienes, de suministro de contenidos digitales y de prestación de servicios relacionados a distancia, sobre todo en línea, cuando así lo acuerden las partes contratantes": Resolución legislativa del Parlamento Europeo, del 26 de febrero de 2014, sobre la propuesta de Reglamento del Parlamento Europeo y del Consejo relativo a una Normativa común de compraventa europea (сом(20I I)0635 - C7-0329/20 I I - 20 I I/O284(COD)) (Procedimiento legislativo ordinario: primera lectura), disponible en: http://www.europarl.europa.eu/sides/ge-

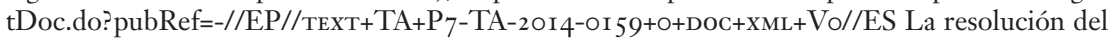
Parlamento es provisional también porque requiere retoques, ya que la versión francesa publicada olvida añadir al Anexo I el inciso "a distancia, sobre todo en línea," presente en las versiones de otros idiomas, como inglés, español o italiano.

i 8 La referencia es a la Comunicación de la Comisión al Consejo y al Parlamento Europeo de I I de julio de 200 I sobre el derecho contractual europeo сом(200I) 398 final, DocE, C 255, de I 3 de septiembre de 200 . Ver, para un análisis estadístico y un elenco de todas las respuestas presentadas a esta: http://ec.europa.eu/consumers/archive/cons_int/safe_shop/fair_bus_pract/ cont_law/comments/summaries/sum_es.pdf LANDo, O. y von BAR, C., "Communication on European Contract Law: Joint Response of the Commission on European Contract Law and the Study Group on a European Civil Code", en Europa e Diritto Privato, n. ${ }^{\circ}$ I, 2002, I33-200; p. ej., Stadenmayer, D., "The Commission Communication on European Contract Law: What 
tual privado" "i9 y que, por otro, defendía la "elaboración y adopción de un corpus de reglas sobre el Derecho contractual ${ }^{\circ}$ de la Unión Europea que tenga en cuenta las soluciones y los conceptos jurídicos comunes". Por ello, aunque se quiere "aproximar el derecho civil y mercantil" ${ }_{21}$ y distinguir "entre las disposiciones jurídicas aplicables al sector empresarial y las aplicables a las relaciones entre empresas y consumidores", al tiempo que se requirió "que las separe sistemáticamente"22, el resultado es limitado. Solamente se ha logrado crear una última versión de un propuesta de reglamento de compraventa que deja clara la distinción y reglamentación de los contratos $\mathrm{B}_{2} \mathrm{~B}$ y $\mathrm{B}_{2} \mathrm{C}$, incorporando la normativa sobre consumidor en un corpus de naturaleza más amplia y no mediante una ley especial $a d$ hoc, aspecto que en estos años -mediante la incorporación de las directivas comunitarias- ha justificado la creación de un código de consumo en algunos países, como Italia o Francia.

future for European Contract Law", European Review of Private Law, 2002, 2, 25 I; AlpA, G., "La Comunicazione n. 398/200 I/CE sulla armonizzazione del diritto privato. Una premessa al dibattito", en Nuova giurisprudenza civile commentata, n. ${ }^{\circ}$ 2, 2002, 42 5-426: Campuzano Díaz, B., “¿Hacia un Derecho Contractual Europeo?”, Anuario de Derecho Europeo, 2002, n. . 2, 54-65.

I9 Esto -dice el Parlamento- no obstante el mandato otorgado por el Consejo Europeo de Tampere. Efectivamente, si el Consejo Europeo de Tampere de 1999 era de una visión más amplia, debe destacarse que la aproximación a que hace referencia la Comisión concierne a una "mayor convergencia en Derecho civil", concepto que es siempre más amplio que el de derecho contractual, pero que se aleja de la noción más abarcadora de derecho privado, propiciada hasta ahora por el Parlamento: Conclusión de la Presidencia n. ${ }^{\circ} 39$ (apartado vII, "Mayor convergencia en Derecho civil" ): "por lo que respecta al Derecho material, se requiere un estudio global de la necesidad de aproximar las legislaciones de los Estados miembros en materia civil para eliminar los obstáculos al buen funcionamiento de los procedimientos civiles. El Consejo debería informar antes de finales de 200 I". Disponible en: http://www.europarl.europa.eu/summits/ tam_es.htm

20 La Resolución del Parlamento Europeo de 15 de noviembre de 200I, expresamente, busca alcanzar soluciones y conceptos jurídicos comunes en estos ámbitos: derecho contractual general, derecho de compraventa, derecho de contratos de servicios, incluidos los servicios financieros y los contratos de seguros, derecho de garantías personales y derecho de obligaciones no contractuales (comisión de delitos, enriquecimiento sin causa), derecho de transmisión de propiedades en el caso de bienes muebles, derecho de garantías crediticias y bienes muebles, derecho de trust. Resolución del Parlamento Europeo, de 15 de noviembre 200I, sobre la aproximación del derecho civil y mercantil de los Estados miembros (сом(200I) 398 - C5-047 I/200I - 2001/2 I87(COS)) Doce C I40 E, de I3 de junio 2002, 538-542.

2 I Resolución del Parlamento Europeo de I 5 de noviembre de 200 I, cit.; ver Arroyo i Amayuelas, E. y Vaquer Aloy, A., "Un nuevo impulso para el derecho privado europeo?", La Ley, I4 de febrero de $200 \mathrm{I}$, n. $^{\circ} 5482$.

22 Resolución, de 23 de marzo de 2006 , sobre el Derecho contractual europeo y revisión del acervo: perspectivas para el futuro (2005/2022(INI)) DOCE C $292 \mathrm{E}$ de I de diciembre de 2006, I09. 
Por ello la reticencia ${ }^{23}$ a la codificación europea, que en el momento de su publicación se consideró "un tanto quijotesca" 24 y cuya perseverancia se ha convertido en "diabólica" 25 (no sólo por el hecho de perseverar ${ }^{26}$, sino porque se ha querido hacerla desaparecer ${ }^{27}$ ), debería en cambio definirse como profética ${ }^{28}$. Por lo tanto, lo que pretendía ser un nuevo codex universal o el nuevo derecho privado europeo, sólo ha llegado, con la CEsL, a ser un probable ${ }^{29}$ texto de derecho internacional (eso sí, privado).

La CISG, que podía verse enfrentada a un posible competidor -es decir, la Propuesta de Reglamento del Parlamento Europeo y del Consejo relativo a una normativa común de compraventa europea-, de momento gana la batalla y actualmente sigue triunfante. De esta manera, el Parlamento Europeo consigue otorgar la debida importancia a la CISG, instrumento que él mismo ya había considerado que podría "constituir en el futuro un fundamento jurídico común, aunque no resolverá por completo el problema de las relaciones jurídicas transfronterizas" ${ }^{\circ}$.

El "réquiem" final a la Common European Sales Law parece darse por parte de la Comisión Europea el r 6 diciembre de 20I4. El programa de trabajo de la Comisión para 20I5, titulado "Un nuevo comienzo" ${ }^{\mathrm{I}}$, en su anexo II elimina el texto de las políticas europeas. Ahora bien, esta eliminación deja unas cenizas

23 La referencia es a Legrand, P., "Against a European Civil Code”, Modern Law Review, i 997, vol. 6o, 44-63. Ver también Legrand P., "Sens et non-sens d'un Code civil européen, Revue internationale de droit comparé, I996, v. 48, n. ${ }^{\circ}$, 779-8 I 2.

24 Así Schepel, H., "Professorenrecht? Le champ du droit privé européen", en Critique Internationale, n. ${ }^{\circ} 6,2005, \mathrm{I} 47$.

25 La referencia es al dicho atribuido a Lucius AnNeus SENECA por el cual "errare humanum est, sed perseverare diabolicum". LEGRAND confirmó su postura años después con un segundo escrito: LEgrand, P., "A diabolical idea", en Aa.vv., Towards a European Civil code, Hartkamp, A. et al. (eds.), Third fully revised and expanded edition, The Hague, Kluwer Law International; Nijmegen, Ars Aequi Libri, 2004, 245-2 22.

26 Efectivamente el escrito "A diabolical idea" de 2004 retoma el escrito de 1997 ("Against a European Civil Code", cit.).

27 La contribución de LEgRAND aparece exclusivamente en la edición de 2004, siendo eliminada en las posteriores.

28 La crítica de LEGRAND esencialmente toca seis puntos: "the idea of European civil code is 'arrogant', 'misleading', 'utopian', 'retrigade', 'the calims in favour of a European Civil Code are woefully under-theorised and reflect an ideological dogmatism devoid of almost any merit a scholarship' [...] 'the contravenes the letter and-rather more importantly-the ethos of European Community law (the Treaty of Rome itself accepts the presence of differences across legal systems within Member States)": LAGRAND, "A diabolical idea", cit., en particular 255 ss.

29 Es probable en cuanto instrumento optativo.

30 Junto al Convenio de Roma sobre la ley aplicable a las obligaciones contractuales según cuanto afirma la Resolución del Parlamento Europeo de I 5 de noviembre de 200 I "sobre la aproximación del Derecho Civil y Mercantil de los Estados miembros”, сом(200 I) 398.

3 I Programa de Trabajo de la Comisión para 20I5 - "Un nuevo comienzo", сом(20I4) 9 Io final, disponible en: http://ec.europa.eu/atwork/pdf/cwp_20I5_es.pdf 
calientes. Efectivamente se prevé una nueva restructuración del proyecto unificador dado que la razón de la retirada es la evolución del producto inicial. Una enésima evolución que incluso parecería aún "más ambiciosa" $3^{2}$ ya que la propuesta de reglamento sobre compraventa viene "modificada para liberar plenamente el potencial del comercio electrónico en el mercado único digital"33. Lo que no se ha conseguido hasta ahora se intentará realizar mediante la creación de un mercado único digital ${ }^{34}$.

Todavía no se sabe el quid 35 de este proceso, que puede ser una simple trasformación o algo más trascendental. ¿Quimera o Fénix?

\section{II. ¿C-ISG O CI-SG? O ¿CESL O CL-ES?}

La Convención de las Naciones Unidas sobre Compraventa Internacional de Mercaderías del I I de abril de i 980 ${ }^{[36]}$ (The United Nations Convention on Contracts for the International Sale of Goods) es normalmente conocida o citada como "Convención de Viena", cuyo acrónimo inglés es CISG. En la casi totalidad de los casos la terminología originaria ha sido abandonada a raíz de su extensión.

Un lector no especialmente entendido en la materia podría interpretar el acrónimo de dos maneras: a) como CI-SG o b) como C-ISG, dependiendo de donde quiera hacer predominar la "I" de International. En el primer caso concluiría que lo internacional es la Convención (CI: International Convention) que regula el contrato de compraventa (SG: Sale of Good). Esta interpretación sería intuitiva ya que las convenciones de esta importancia son a menudo internacionales, pero desconocería así que en inglés el atributo debe colocarse antes del nombre.

32 La Comisión en su "nuevo comienzo" afirma que "[e]n algunos casos, la Comisión propone retirar propuestas para sustituirlas por otras más ambiciosas o para adaptarlas mejor a sus diez prioridades". Si la razón fuera otra se estaría reconociendo expresamente el fracaso original del proyecto, ya que en el nuevo programa de trabajo se evidencian otras razones para el retiro que tienen todas una visión negativa. La Comisión evidencia que se han retirado las propuestas (indicadas en el Anexo II) que han permanecido "estancadas en la mesa de negociación", "superadas por los acontecimientos", o que han quedado "desvirtuadas hasta tal punto que ya no pueden alcanzar su objetivo inicial": Programa de Trabajo de la Comisión para 20 5, "Un nuevo comienzo", cit., 4 .

33 Anexo II, I4 del Programa de Trabajo de la Comisión para 2015. "Un nuevo comienzo",

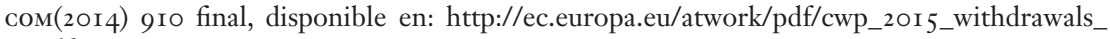
es.pdf.

34 Ver Programa de Trabajo de la Comisión para 2015 - "Un nuevo comienzo", cit., 7.

35 El inicio de este nuevo proceso se ha inaugurado con dos propuestas de directivas, ambas del 9 de diciembre 2015: I) Propuesta de Directiva del Parlamento Europeo y del Consejo relativa a determinados aspectos de los contratos de suministro de contenidos digitales: $\operatorname{com}(2015) 634$ final; 2) Propuesta de Directiva del Parlamento Europeo y del Consejo sobre contratos en línea y otras ventas a distancia de bienes muebles: $\operatorname{com}(2015) 635$ final (addenda: 3 de marzo de 20 16).

36 Instrumento de adhesión por parte de España de I 7 de julio de I990 (вов n. ${ }^{\circ}$ 26/ I 99 I, de 30 de enero; rect. воЕ n. ${ }^{\circ}$ 282/I 996 , de 22 de noviembre de I996). 
Por ello, es la interpretación b) la correcta, es decir, la que identifica que existe una convención (C: Convention) relativa a la compraventa internacional de mercaderías (ISG: International Sale of Goods). Según esta segunda interpretación (C-ISG: Convention on... International Sale of Goods) -que refleja la realidad en el caso concreto-, se evidencia que se trata de un texto de derecho internacional privado (y no de derecho común) que se aplica a las compraventas trasfronterizas, que se celebran entre los Estados: por lo tanto no hay lugar a dudas sobre que se habla de la compraventa internacional de mercaderías entre los Estados que han firmado el Convenio.

Desde una perspectiva sustancial la diferencia interpretativa no es irrelevante ya que según la primera interpretación podría estar haciéndose referencia a un texto unificador al que se someten muchos países, con una regulación común sobre la compraventa: un texto que regula de manera común el contrato de cualquier compraventa en los ordenamientos jurídicos de los Estados adheridos al Convenio; un texto, en definitiva, que ha uniformado el contrato de compraventa en estos Estados.

Todos los razonamientos efectuados hasta ahora parecerían sobrar al resultar casi obvios, y un lector o jurista que interpretara el texto anterior de manera inapropiada -según la interpretación a) arriba planteada- debería indudablemente rectificar su visión. Ahora bien, si en este error cayera el legislador quizás la circunstancia sería más reprochable, y así lo es.

La propuesta de reglamento que ha surgido del proceso de unificación del derecho privado es relativa a la Common European Sales Law, conocida con su acrónimo de CESL, ya que el proyecto inicial era crear un derecho común europeo sobre la compraventa. Sin embargo, en su versión oficial española, el título de la propuesta de reglamento no se refiere a un "derecho común europeo sobre la compraventa" -como lo hacen en cambio las otras versiones oficiales ${ }^{37}$ - sino a una "Normativa común de compraventa europea". ¿CESL o CL-ES? El error del legislador europeo en la versión española parece a todas luces evidente. ¿ $\mathrm{O}$ acaso este error se habrá convertido en un acierto? A pesar del nomen iuris diferente, el contenido de la propuesta de reglamento es el mismo. Sin embargo, dado que el documento ha sido traducido a 24 idiomas de la Unión Europea, cabe preguntarse: ¿hay diferencia entre hablar de un "derecho común europeo" relativo a la compraventa y hablar de una "normativa común" de "compraventa europea"?

Efectivamente la idea originaria era la unificación, pero se ha visto que aunque se quiso unificar Europa y su normativa creándose un "derecho común europeo" relativo a determinados contractos (aunque se limitó al de compraventa), finalmente en su última versión del texto se ha contado con un instrumento

37 Las versiones francesa, inglesa, italiana, alemana y portuguesa hablan, respectivamente, de "droit commun européen de la vente", "Common European Sales Law", "diritto comune europeo della vendita", "Gemeinsames Europäisches Kaufrecht" y "direito europeu comum da compra e venda". 
(optativo) de derecho internacional privado que se aplica sólo a la "compraventa europea" -es decir, regulada con una "normativa común” a condición de que sea trasfronteriza, europea. Esto es, en los Estados miembros, salvaguardando las tipologías propias e internas de cada ordenamiento nacional.

Antes he afirmado que en el proceso de unificación del derecho privado europeo, ni siquiera el mismo legislador-autor sabe todavía qué producto ha creado. Aclarar esta cuestión no es fácil ya que se encuentran involucradas no solamente variables jurídicas sino políticas, sociales e idiomáticas. Así que, antes de asumir que la tendencia se dirige verdaderamente hacia un "Código europeo común de Derecho privado", deberíamos preguntarnos si lo que realmente queremos llevar a cabo es una tarea unificadora, en la que los Estados miembros se someterán al mismo texto legal, o si más bien lo que quiere realizarse es una misión uniformadora y armonizadora. Pero todavía se desconoce si se quiere uniformar, armonizar o unificar.

En Italia, la eliminación del Código de Comercio mediante la aprobación del codice civile de $194^{2}$ ha conducido, como destacó en su día la doctrina ${ }^{3}$, a que ya no pueda hablarse de una diferenciación entre derecho privado y derecho civil, en tanto que relación entre género y especie. Por ello, la mirada atenta sabe que lo que todos conocen como codice civile italiano, desde r942, nunca lo ha sido, ya que más bien es un codice di diritto privato, que contiene una normativa tanto de derecho civil como mercantil y que adopta un concepto de "derecho civil unificador"39. Aquí la máxima latina del "nomen iuris" encuentra su mayor y emblemático botón de muestra, y en el caso de la propuesta de reglamento europeo -así como están las cosas- haría inclinar la balanza a favor de que efectivamente se trata de una "normativa común de compraventa europea”, en cuanto tiene naturaleza de instrumento de derecho internacional privado. Por ello, el claro error en la versión oficial española de la propuesta de reglamento ha sido un acierto. Lo que es verdad es que en este último contexto se sufre una ambigüedad como aquella de la metamorfosis kafkiana ${ }^{40}$, que no sabemos todavía a qué conducirá. ¿Cómo será el nuevo mercado único digital4?

$3^{8}$ Nicolò, R., (voz) "Diritto civile", en Enciclopedia del Diritto, xiI, Milano, i964, 906.

39 Cian, G., "Il diritto civile come diritto privato comune", en AA.vv., La civilistica italiana dagli anni '5o ad oggi, Congresso dei civilisti italiani, Venezia, 23-26 giugno i 989, Cedam, Padova, I99I, I I.

40 El título original que el célebre autor austrohúngaro dio a su obra fue Die Verwandlung, sin hacer uso de la palabra "Methamorphose"; sin embargo, la obra en el resto del mundo es conocida por la utilización de la palabra que algunos traductores consideraron más apropiada al contenido del texto.

4I El legislador europeo parece haber tomado partido decidiendo elegir como instrumento dos (propuestas de) directivas y no de reglamento (addenda: 3 de marzo de 2016). 


\section{La tendencia de los proyectos de unificación normativa hacia la internacionalización privatista}

Es preciso destacar que los Principios UnIDroit y la CISG, además de importantes e indiscutidos actores en el panorama jurídico internacional, son instrumentos de derecho internacional privado y los dos se relacionan con proyectos más amplios de unificación del derecho. Si la cIsG contribuyó a hacer desaparecer uno de dichos proyectos, los Principios Unidroit son sólo la primera etapa de un recorrido más largo aún no culminado.

\section{A. Los Principios UnIDroIT}

El Instituto Internacional para la Unificación del Derecho Privado, conocido como Unidroit, nació en I926 para "para examinar la manera de armonizar y coordinar el Derecho privado de los Estados y de los grupos de Estados y preparar gradualmente la adopción por parte de los diversos Estados de normas uniformes de Derecho privado" 42 .

El Instituto es consciente de que para lograr este objetivo es preciso comenzar armonizando el derecho privado internacional, dedicando una especial atención a las obligaciones y contratos. Fruto de este proceso, iniciado en I970 ${ }^{[43]}$, son las tres ediciones de los Principios Unidroit. Sin embargo, el recorrido de Unidroit se encuentra aún en su fase inicial y, de momento, es de carácter limitado en un triple sentido, ya que se circunscribe únicamente al derecho mercantil, es aplicable sólo por voluntad de las partes y se refiere a los contratos internacionales.

\section{B. La unificación del derecho latinoamericano y la Convención de Viena}

Como dije páginas atrás, en la Unión Europea se está produciendo un fenómeno de carácter patológico, como es la trasformación de un proyecto inicialmente dirigido a la creación de un derecho común en un instrumento de derecho

42 Art. I Estatuto fundacional Unidroit.

43 Así se declaraba expresamente en una circular de i970: "nuestro Instituto se ha propuesto, en el cuadro de su programa de trabajo y paralelamente a sus esfuerzos de unificación referentes a materias especiales, proceder al estudio de las perspectivas de una codificación progresiva del derecho del comercio internacional. Un estudio preliminar efectuado al respecto, lleva a la conclusión de que la codificación debe ser encarada de una manera gradual y que la primera tarea en el cuadro de un plan general y orgánico de unificación, debería consistir en preparar un proyecto concerniente a la parte general del Derecho de Obligaciones, cuyos principios uniformes estarán destinados a constituir la base de la codificación progresiva del derecho del comercio internacional": Unidroit, Circular M.308/IV-A44, 4 de diciembre de i970, Secretario General Mario Matteucci. 
internacional privado. Este fenómeno muestra un evidente paralelismo con lo ocurrido en América Latina hace ya unas décadas.

En efecto, el así llamado "Panamericanismo"44 pretendió en su día unificar el derecho privado, aunque -como se verá- sin mucho éxito, ya que el objetivo unificador quedó pronto arrumbado y dejó paso enseguida a un proceso de creación de normas de derecho internacional privado, si bien es cierto que en tiempos recientes, como explicaré más adelante, se ha vuelto a retomar aquel objetivo unificador. Efectivamente, el proyecto de "Código Civil Americano Único”, mediante un estudio comparativo del Código Civil de Brasil con los demás códigos civiles latinoamericanos impulsado desde $1933^{[45]}$, y la más ambiciosa codificación del derecho privado en todo el continente americano tratando de unificar tanto el derecho civil como el mercantil - auspiciada cinco años más tarde ${ }^{46}-$, se quedaron en nada. De poco sirvió la creación de una Comisión Per-

44 Actualmente existe la clara voluntad política de unificar la legislación de América Latina con un carácter más amplio que el exclusivamente relativo a las obligaciones y contratos mediante leyes marco. Muchos son los proyectos de leyes marco aprobados por el Parlamento para su consideración en los Congresos latinoamericanos. Pueden consultarse en: www.parlatino.org/ temas-especiales/proyectos-de-leyes-marco.html [Última fecha de consulta: i 3 de marzo de 2015].

45 Así, en la séptima Conferencia internacional americana de 1933 se quiere "emprender un estudio comparado del Código Civil del Brasil con sus respectivos Códigos Civiles, al efecto de que la próxima Conferencia Internacional Americana esté en aptitud de enviar esos trabajos a una comisión de juristas que emprenda la obra del Código Civil Americano Único". Del mismo modo, expresamente en el punto xxi, "Unificación del Derecho Civil”, de la Resolución de la séptima Conferencia internacional americana (Montevideo, 3 a 26 de diciembre de I933), punto aprobado el 22 de diciembre de I933, como se destaca de la documentación oficial, en Conferencias Internacionales Americanas (I889-1936). Recopilación de los tratados y mociones adoptadas por las siete primeras Conferencias Internacionales Americanas, la Conferencia Internacional Americana de Conciliación y Arbitraje y la Conferencia Interamericana de Consolidación de la Paz, con varios documentos relativos a la organización de las referidas Conferencias, prefacio de Leo S. Rowe, introducción de James Brown Scotт, Dotación Carnegie para la Paz Internacional, Washington, I 938, 494. Debe destacarse que incluso el Código Bustamante de 1928 , texto más bien adscribible al derecho internacional privado, contenía un título cuarto de "Obligaciones y Contratos": ver arts. I64-I 74 por lo que respecta a las obligaciones y arts. I 75-I 86 por lo que respecta a los contratos.

46 Así, en la Conferencia de I 938 se afirmaba que era "aspiración americana propender a la unificación de las normas positivas del Derecho Privado en el Continente, bajo la forma codificada". Esto por cuanto se considera una "uniformidad de los derechos comercial y civil". Así se titula el punto vir: "Unificación del Derecho Civil", de la Resolución de la octava Conferencia internacional americana (Lima, 9 a 27 de diciembre de I938), punto aprobado el 2 I de diciembre de i 938. Ver Conferencias Internacionales Americanas, Primer Suplemento (1938-1942). Convención, declaración, recomendaciones y mociones adoptadas por la octava Conferencia Internacional Americana y por las reuniones primera, segunda y tercera de consulta de los Ministros de Relaciones Exteriores de las Repúblicas Americanas respectivamente en Panamá, La Habana y Río de faneiro; así como el texto de documentos tocantes a la organización de dichas Conferencias y datos sobre conferencias, comisiones $u$ otras entidades técnicas interamericanas, División de Derecho Internacional de la Dotación para la Paz Internacional, nota preliminar de Alberto Lleras, introducción de George A. Finch, Dotación Carnegie para la Paz Internacional, Washington, I943, 24-26. 
manente de Juristas 47 , formada por un miembro designado por Estados Unidos y otros dos latinoamericanos $4^{8}$, con la función de conciliar mejor el common law con el derecho continental latinoamericano. En efecto, como se ha destacado49, las circunstancias de la época impidieron alcanzar las finalidades perseguidas. Desde ese momento el propósito de unificar mediante una normativa común se trasforma en un proyecto más bien tendiente a buscar soluciones de derecho internacional privado.

Desde su creación, en I948 ${ }^{[50]}$, la Organización de los Estados Americanos dedica una atención preferente al derecho internacional privado, mientras que el interés por la unificación del derecho privado y civil se circunscribe más bien a la compraventa, en completo paralelismo con la evolución experimentada durante los últimos años en el ámbito europeo y que ha dado lugar a la CESL.

Es en el referido contexto donde hay que enmarcar el fracaso de dos proyectos sobre la compraventa de bienes muebles -el primero elaborado en $1953^{\left[5^{\mathrm{I}}\right]} \mathrm{y}$ el segundo en $1965^{\left[5^{2}\right]}$ así como el abandono del ambicioso objetivo de alcanzar una unificación que comprendiese todo el continente americano. Hay que tener en cuenta, además, que la aprobación del Uniform Commercial Code53 contribuyó de forma decisiva a poner fin a los esfuerzos de unificación con Estados Unidos.

Sobre su composición ver el punto vir de la Resolución de la octava Conferencia internacional americana y sus actas, en Conferencias Internacionales Americanas, Primer Suplemento (1938-1942), cit., $24-26$ y 438 y 439 .

48 Estos fueron sorteados entre los veinte propuestos por cada uno de los países miembros, resultando ser uno de Perú y otro de Venezuela.

49 Como señala Parra-Aranguren, G., "La importancia del Instituto Internacional para la Unificación del Derecho Privado (UNIDroit) en la futura uniformidad jurídica del hemisferio americano", Revista de la Facultad de Ciencias furídicas y Políticas (Caracas), n. . 86, I992, 40; ver también 64 y 65 .

50 En estos términos Parra-Aranguren, ob. cit., 40; ver también 64 y 65 .

$5^{\text {I } ~ " R e g l a s ~ U n i f o r m e s ~ s o b r e ~ v e n t a ~ d e ~ b i e n e s " . ~ E l ~ c o m i t e ́ ~ j u r i ́ d i c o ~ e l a b o r o ́ ~ u n ~ " P r o y e c t o ~ p r o v i s i o-~}$ nal" no aprobado por el Consejo Interamericano de Jurisconsultos en su segunda Reunión de 1953: "Segunda Reunión del Consejo Interamericano de Jurisconsultos, Buenos Aires, 20 de abril a 9 de mayo de 1953", en Actas y Documentos, v. I, Washington, I953, I38-I49.

52 En "Convención de Ley uniforme de venta internacional de bienes muebles corporales", elaborado por el Comité Jurídico Interamericano y rechazado en la quinta reunión por el Consejo Interamericano de Jurisconsultos celebrada en El Salvador en 1965: ver Parra-Aranguren, ob. cit., 66.

53 El Uniform Commercial Code de Estados Unidos nació esencialmente por iniciativa privada y no parlamentaria, y es fruto de la National Conference of Commissioners on Uniform State Law (NcCULS, que desde 2007 es llamada Uniform Law Commission) y el American Law Institute (ALI). Ver American Law Institute, National Conference of Commissioners on Uniform State Laws, Uniform Commercial Code: Official text and comments; 2013-2014 Edition, Thomson Reuters, 2013 .

Para un breve estudio preliminar en español sobre el Uniform Commercial Code y su versión oficial actualizada hasta el año 2004 en este idioma, ver Garrido, J. M., Código Uniforme de Comercio de los Estados Unidos. Texto oficial. Versión en castellano autorizada por The American Law Institute, trad. y estudio preliminar de J. M. Garrido, Madrid, Marcial Pons, 2002. 
Por si fuera poco, otro instrumento -precisamente de derecho internacional- vino a jugar un papel clave para cortar las alas a los proyectos dirigidos a conformar un derecho común. Efectivamente, mientras los trabajos para la elaboración de un proyecto latinoamericano no conseguían avanzar o lo hacían lastrados con interminables dilaciones, la aparición de un nuevo instrumento -la Ley Uniforme sobre la Venta Internacional de Objetos Mobiliarios Corporales (ULIS) de I964 ${ }^{[54]}$ - acabó por frustrar definitivamente los esfuerzos para hacer realidad dicho proyecto común latinoamericano. En I967, a los pocos años de aprobarse la ULIs, en efecto, parece constatarse un generalizado estado de opinión que mantiene que el nuevo texto "constituye un documento que satisface las necesidades de los países americanos en la materia" 55 .

La Convención de Viena derroca definitivamente todo proyecto de unificación en Latinoamérica. Por ello, en las primeras dos conferencias interamericanas de derecho internacional privado (en I975 y i979) se acogió la recomendación del Comité Jurídico Interamericano del I 3 enero de ${ }_{1977^{\left[5^{6}\right]}}$ en el sentido de abstenerse de estudiar el tema, ya que la Comisión de las Naciones Unidas para el Derecho Mercantil Internacional estaba elaborando mejoras a la ulis que acabaron cristalizándose en la Convención de Viena.

Por lo tanto, lo que había nacido como un ambicioso proyecto de unificar el derecho americano vino a disgregarse, por un lado, con la escisión de Estados Unidos -que consagra su unificación legislativa en este ámbito con el Uniform Commercial Code desde I952-, y por otro, con la focalización en una compraventa que eleva la Convención de Viena como punto de referencia también para América Latina 57 . Por todo ello, tal como está ocurriendo actualmente en la realidad de la Unión Europea, un procedimiento de más amplio alcance y relativo a todo el derecho civil se ha contraído a la regulación de una específica tipología

Elaborada en Conferencia diplomática sobre la unificación del derecho en materia de venta internacional de bienes muebles.

55 Como bien destaca Parra-Aranguren, el Congreso, el 2 I de septiembre de i967, declaró: "que no existen motivos para propiciar la vigencia de un documento regional que regule la venta internacional de bienes muebles, b) que la Ley Uniforme sobre venta internacional de bienes muebles aprobada por la conferencia diplomática de La Haya (1964) constituye un documento que satisface las necesidades de los países americanos en la materia”: Parra-Aranguren, ob. cit., 66. Ver García Amador, F. V., Sistema Interamericano a través de Tratados, Convenios y otros documentos, v. I, Asuntos jurídicos políticos, compilación anotada por Francisco V. García Amador, Washington, I981, 427-429.

56 Lo reporta Parra-Aranguren, ob. cit., 66.

57 La Iv Conferencia Interamericana sobre Derecho Internacional Privado (cIPID.Iv), con su segunda resolución, recomienda a los Estados miembros que todavía no son parte de la Convención de Viena, incorporarla: Acta final, Cuarta Conferencia Especializada Interamericana sobre Derecho Internacional Privado, Montevideo, 9 a I 5 de julio de I989, Washington, I990, Io. 
de compraventa, la transfronteriza de bienes muebles, sin unificar el derecho interno de todos los países ${ }^{5}$.

\section{La nueva apuesta en Latinoamérica sobre derechos de contratos}

En noviembre de 2013, con ocasión de un congreso celebrado en Santiago y Valparaíso ${ }^{59}$, se presentó públicamente la primera versión de los Principios latinoamericanos de derecho de contratos ${ }^{60}$. Este proyecto nace de un proyecto patrocinado y auspiciado en conjunto por la Fundación Fernando Fueyo Laneri ${ }^{61}$ y la Fondation pour le Droit Continental, y reúne a varios académicos de universidades de Argentina, Brasil, Colombia, Chile, Francia, Paraguay, Uruguay y Venezuela; a los cuales recientemente se ha añadido Guatemala. Este es uno de los varios proyectos de armonización del derecho latinoamericano emprendidos

$5^{8}$ También es fundamental recordar aquí la existencia de un instrumento complementario a la Convención de Viena, que, sin embargo, no fue adoptado por todos los países que asumieron el primer instrumento. Hablo de la Convención sobre la Prescripción en materia de compraventa internacional de mercadería, aprobada en Nueva York el I4 de junio de I 974 y su protocolo de enmienda del i I de abril de i980; este último, encaminado a armonizar su texto con el de la Convención de las Naciones Unidas sobre los Contratos de Compraventa Internacional de Mercadería. Ver: http://www.uncitral.org/uncitral/es/uncitral_texts/ sale_goods/I974Convention_limitation_period.html

59 Congreso internacional "El Derecho de los contratos: formación, cumplimiento e incumplimiento", celebrado en Santiago de Chile y Valparaíso, los días i9 y 20 de noviembre de 2013 , respectivamente, y patrocinado en conjunto por la Fundación Fernando Fueyo Laneri y las facultades de Derecho de la Universidad Diego Portales y de la Pontificia Universidad Católica de Valparaíso. Sobre el Congreso, ver Rome, F., "Vive l'Amérique latine!", Recueil Dalloz, n. o 4I, 201 3; Morales Moreno, A. M., "Los Principios latinoamericanos de derecho de los contratos: un debate abierto sobre las grandes cuestiones jurídicas de la contratación", Anuario de Derecho civil, t. LVVII, fasc. I, 2014, $227^{-2} 54$.

60 Vid. aA.vv., El Derecho de los contratos en Latinoamérica, Pizarro Wilson, C. (coord.), Santiago y Bogotá, Fundación Fueyo Laneri, Universidad Externado de Colombia y Universidad del Rosario, 20I2. El texto de los principios puede consultarse también como anexo al artículo de Morales Moreno, ob. cit., 227 ss.; para el texto de los Principios en particular, 24I-254; Hondius, E., "Latin America Goes PECL", European Review of Private Law, vol. 2 I, n. ${ }^{\circ}$ 2, 2013 , 419-422, Ferrante, A., "Es correcta la elección del método de cálculo de la reducción del precio en los Principios latinoamericanos de Contratos?”, Revista Chilena de Derecho Privado, n. ${ }^{\circ}$ 22, 20I4, 9-49; sobre el procedimiento de unificación en particular, 9- I9; MомвERG URIBE, R., "Harmonization of Contract Law in Latin America: Past and present initiatives", Uniform Law Review, v. 19, n. ${ }^{\circ} 3,2014,41 \mathrm{I}-428$.

6I En este sentido, la Fundación continúa el esfuerzo de carácter unificador del derecho que ya Fueyo Laneri impulsaba desde una óptica nacional y después latinoamericana: ver, respectivamente, Fueyo Laneri, F., "Reforma del Código Civil Chileno bajo la inspiración de Bello", Revista de Derecho Español y Americano, n. ${ }^{\circ}$ i I, enero-marzo, i 966, 99-i i 9; Fueyo Laneri, F., "Proyecto de Código único de las obligaciones y de los contratos para los países de origen latino", en aA.vv., Le projet franco-italien du Code des obligations, Rotondi, M. (ed.), Padova, Cedam, I980, I97-233. 
en las últimas décadas ${ }^{62}$, y demuestra la voluntad de retomar un discurso interrumpido hace años.

La gran ventaja que presenta Latinoamérica respecto a la Unión Europea, aunque de momento hablamos de proyectos sólo de naturaleza académica, es la predominante homogeneidad lingüística ${ }^{6} 3$. Efectivamente, aun cuando tanto el Parlamento Latinoamericano como la Unión Europea poseen dos idiomas oficiales (respectivamente, español y portugués e inglés y francés), la realidad en cada ámbito es totalmente diferente ya que la unificación idiomática no es un problema en el primer caso, pues la mayoría de los países tienen el castellano como lengua principal ${ }^{64}$. El Proyecto está en plena fase de desarrollo ${ }^{65}$ y perfeccionamiento $^{66}$, y es previsible que no tardará en cosechar nuevos resultados ${ }^{67}$,

62 Para una panorámica y una reconstrucción de estos proyectos ver FERRANTE, ob. cit., i 8 ss.

63 Aspecto que no se da en cambio en la Unión Europea, ya que de los 28 países miembros, solamente en seis se hablan los idiomas oficiales (Francia, Irlanda, Luxemburgo, Malta, Países Bajos y Reino Unido).

64 De los 23 países miembros sólo cinco no lo hablan: Surinam, Curazao (neerlandés), Aruba (inglés) y Sint Maarten (neerlandés e inglés) y Brasil (que sin embargo habla el otro idioma oficial, el portugués).

65 Ya la doctrina ha empezado a tratar temas específicos de los principios, por ejemplo en relación con la buena fe, la reducción del precio, el error, el incumplimiento contractual y la lesión: ver Eyzaguirre Baeza, C. y Rodríguez Díez, J., "Expansión y límites de la buena fe objetiva - A propósito del Proyecto de Principios latinoamericanos de los contratos", Revista Chilena de Derecho Privado, n. ${ }^{\circ}$ I , 20 I3, I37-2 I 6; Ferrante, ob. cit.; De La Maza Gazmuri, I., "El error en los Principios latinoamericanos de derecho de los contratos", en Antoni Vaquer Aloy, Esteve Bosch Capdevila y María Paz Sánchez González (eds. lits.), El derecho común europeo de la compraventa y la modernización del derecho de contratos, Barcelona, Atelier, 20 I 5, 789-798; VIDAL Olivares, Á. R. "Incumplimiento contractual y pretensión de cumplimiento específico en los principios latinoamericanos de derecho de contratos (PLDc)", ibíd., 745-765; López Díaz, P. V., "Por una noción amplia de lesión en el Código Civil chileno: una relectura a partir del principio de equilibrio contractual y la idea de excesiva desproporción contenida en el borrador de los Principios latinoamericanos de contratos", AA.vv., Estudios de Derecho Civil x, Álvaro VIDAL Olivares, Gustavo Severín Fuster, Claudia Mejías Alonzo (coords.), Thomson Reuters, Santiago de Chile, 2015, 699 -723. Más en general, ver también Oviedo Albán, J., "La Convención sobre compraventa internacional y la modernización del derecho latinoamericano de contratos: un tema pendiente”, en Vaquer Aloy, Bosch Capdevila y Sánchez González (eds. lits.), ob. cit., 769-788.

66 Debe evidenciarse que en el mes de junio se ha celebrado en Oxford un encuentro para presentar y discutir los Principios Latinoamericanos de Derecho de los Contratos (PLDC). Las actas están en prensa en la colección Studies of the Oxford Institute of European and Comparative Law (Hart Publishing). Para un informe de la jornada ver Morales Ortiz, María Elisa, "Informe sobre Conferencia internacional 'The Future of Contract Law in Latin America'", Revista de Derecho Civil (España), n. ${ }^{\circ}$ 3, 20I 5, 22 I-225; Gregoraci Fernández, B., “The Future of Contract Law in Latin America', 25 de junio de 20 I 5 , Institute of European and Comparative Law, University of Oxford (Reino Unido)", Anuario de Derecho Civil, n. ${ }^{\circ}$ 3, 201 5, ro83-iogo. La última reunión del Grupo se ha celebrado en Bogotá, en la Universidad Externado de Colombia (27 y 28 de octubre de 20 I 5) y en la del Rosario (29 de octubre de 2015).

67 El interés de la academia española es evidente ya que la Real Academia Española de Legislación y Jurisprudencia realizará los próximos días i 6 y i 7 de junio, en conjunto con la Universidad Autónoma de Madrid, las “Jornadas de Discusión y Análisis de los Principios latinoamericanos 
cuya plasmación en la realidad no cabe sino esperar con anhelo. ¿Será esta la nueva Fénix del Panamericanismo? Seguramente este nuevo proyecto podría imponerse como importante instrumento de soft law de referencia para Latinoamérica.

Adicionalmente otro grupo, el GADAL -Grupo para la Armonización del Derecho en América Latina ${ }^{68}$ - está sumando esfuerzos para la unificación del derecho de obligaciones en América Latina, que encuentra sus antecedentes inmediatos en el Grupo para la Armonización del Derecho Privado Latinoamericano $^{69}$. En cualquier caso, todo proyecto que se emprenda en este campo habrá de tomar desde el inicio claro partido por una de las tres posibles opciones: uniformar, armonizar o más bien unificar ${ }^{70}$. En efecto, las soluciones serán diferentes en función de la elección adoptada en cada caso. Y según cuál sea la opción de partida tomada, el resultado tenderá hacia la elaboración de un instrumento de derecho internacional o hacia la formación de un derecho propiamente común, sin fronteras.

\section{Bibliografía}

Aa.vv., Terminologie contractuelle commune - Projet de cadre commun de référence, Société de Législation Comparée et Association Henri Capitant (ed.), coll. "Droit privé comparé et européen”, v. 6, Munich, Sellier, 2008.

AA.vv., Principes contractuels communes - Projet de cadre commun de référence, Société de Législation Comparée et Association Henri Capitant (ed.), coll. “Droit privé comparé et européen”, v. 7, Munich, Sellier, 2008.

de Derechos de contratos". Estas jornadas tienen como finalidad participar en el proceso de discusión de diferentes secciones de los Principios.

68 El Grupo hasta ahora se ha reunido en Lima (2013), México y Bogotá (2014), y su cuarto encuentro se ha celebrado en la Universidad de Concepción, Santiago de Chile, los días 9 a i de diciembre; tiene previstas sus próximas reuniones en México y Perú a lo largo del año 20r6. La primera publicación del Grupo, fruto de la reunión de México, es: Grupo para la Armonización del Derecho en América Latina, Pacta sunt servanda y rebus sic stantibus: desarrollos actuales $y$ perspectivas históricas, Carlos Soriano Cienfuegos (ed.), México, Universidad PanamericanaNovum, 20I4. El Grupo, por el momento, está integrado por juristas de siete países (Argentina, Brasil, Colombia, Chile, México, Perú y Venezuela); para mayor informaciones sobre el Grupo véase: http://gadal.simplesite.com/ o en http://gadal.uexternado.edu.co/ [última consulta: I3 de octubre de 2015].

69 Sobre este grupo, con reenvíos bibliográficos, ver FerRanTe, ob. cit., i 8 y ss.

70 Sobre los matices entre estas tres terminologías ver Jeammaud, A., "Unification, uniformisation, harmonisation: de quoi s'agit-il?”, en Osman, F. (dir.), Vers un Code européen de la consommation? Codification, unification et harmonisation du droit des Etats-membres de l'Union européenne: Actes et débats de Colloque: Lyon, les I2 et I3 décembre 1997, Bruylant, Bruxelles, I998, 35-55; Osman, F., "Codification, unification, harmonisation du droit en Europe: un rêve en passe de devenir réalité", ibíd., I I-34. 
AA.vv., European Contract Law. Materials for a Common Frame of Reference: Terminology. Guiding Principle, Model Rules, Société de LÉgislation Comparée et Association Henri Capitant (ed.), Bénédicte Fauvarque-Cosson y Denis Mazeaud (coords.), Munich, Sellier, 2008.

aA.vv., El derecho de los contratos en Latinoamérica, Pizarro Wilson, C. (coord.), Santiago y Bogotá, Fundación Fueyo Laneri, Universidad Externado de Colombia y Universidad del Rosario, 20 I 2.

American Law Institute, National Conference of Commissioners on Uniform State Laws, Uniform Commercial Code: Official Text and Comments, 2OI3-2OI4 Edition, Thomson Reuters, 2013.

Arroyo i Amayuelas, E. y Vaquer Aloy, A., "Un nuevo impulso para el derecho privado europeo?", La Ley, I4 de febrero de 200 I, n. ${ }^{\circ} 5482$.

Alpa, Guido, "La comunicazione n. 398/200 I/CE sulla armonizzazione del diritto privato. Una premessa al dibattito", en Nuova giurisprudenza civile commentata, n. ${ }^{\circ} 2,2002,425-426$.

Campuzano Díaz, B., “¿Hacia un derecho contractual europeo?”, Anuario de Derecho Europeo, n. ${ }^{\circ}$ 2, 2002, 54-65.

Cian, G., "Il diritto civile come diritto privato comune", AA.vv., La civilistica italiana dagli anni 'so ad oggi, Congresso dei civilisti italiani, Venezia, 23-26 giugno r989, Cedam, Padova, r991, 9-25.

De La Maza Gazmuri, I., "El error en los principios latinoamericanos de derecho de los contratos”, en Antoni Vaquer Aloy, Esteve Bosch Capdevila y María Paz Sánchez González (eds. lits.), El derecho común europeo de la compraventa y la modernización del derecho de contratos, Barcelona, Atelier, 20 I 5 , 789-798.

Eyzaguirre Baeza, C., y Rodríguez Díez, J., "Expansión y límites de la buena fe objetiva - A propósito del Proyecto de Principios latinoamericanos de los contratos", Revista Chilena de Derecho Privado, n. 2 I, 2013 , I37-2 I6.

Fauvarque-Cosson, B., "Vers un droit commun européen de la vente", Recueil Dalloz, 201 2, 34 . 
Ferrante, A., "Es correcta la elección del método de cálculo de la reducción del precio en los Principios latinoamericanos de Contratos?", Revista Chilena de Derecho Privado, n. ${ }^{\circ}$ 2, $2014,9-49$.

Fueyo Laneri, F., "Reforma del Código Civil chileno bajo la inspiración de Bello", Revista de Derecho Español y Americano, n. ${ }^{\circ}$ I I, enero-marzo, I966, 99I I9.

Fueyo Laneri, F., "Proyecto de código único de las obligaciones y de los contratos para los países de origen latino", en AA.vv., Le projet franco-italien du Code des obligations, Mario Rotondi (ed.), Padova, Cedam, i980, I97-233.

García Amador, F. V., Sistema Interamericano a través de Tratados, Convenios y otros documentos, v. I, Asuntos jurídicos políticos, compilación anotada por Francisco V. García Amador, Washington, I98I.

Garrido, J. M., Código uniforme de comercio de los Estados Unidos. Texto oficial. Versión en castellano autorizada por The American Law Institute, trad. y estudio preliminar de J. M. Garrido, Madrid, Marcial Pons, 2002.

Gregoraci Fernández, B., “'The Future of Contract Law in Latin America', 25 de junio de 2015 , Institute of European and Comparative Law, University of Oxford (Reino Unido)", Anuario de Derecho Civil, n. ${ }^{3}$, 20 I 5, Io83-109o.

Grupo para la Armonización del Derecho en América Latina, Pacta sunt servanda y rebus sic stantibus: desarrollos actuales y perspectivas históricas, CARLOS Soriano Cienfuegos (ed.), México, Universidad Panamericana-Novum, 2014 .

Hondius, E., "Latin America Goes PecL", European Review of Private Law, v. 2 I, n. ${ }^{\circ}$ 2, 2013,4 I $9-422$.

Jeammaud, A., "Unification, uniformisation, harmonisation: de quoi s'agit-il?", en Osman, Filali (dir.), Vers un Code européen de la consommation? Codification, unification et harmonisation du droit des États-membres de l'Union européenne: Actes et débats de Colloque: Lyon, les I 2 et I 3 décembre I997, Bruxelles, Bruylant, I998, 35-55.

Lando, O., "My life as a lawyer", Zeitschrift für Europäisches Privatrecht, n. ${ }^{\text {3 }}$, 2002, 508-522. 
Lando, O., "European Contract Law", The American fournal of Contract Law, 1983, 653-659.

Lando, O. y Beale, H., Principles of European Contract Law. prepared by the Commission on European Contract Law, Ole Lando, Hugh Beale (eds.), Nijhoff, Dordrecht 1995 (Les Principes du droit européen du contrat: l'exécution, l'inexécution et ses suites, version française: Isabelle de Lamberterie, Georges Rouhette, Denis Tallon [trad.]), Paris, La Documentation française, I997).

Lando, O. y Beale, H. (eds.), Principles of European Contract Law. Parts I and II, combined y revised for the Commission on European Contract Law, The Hague, London, Boston, Kluwer Law International, 2000.

Lando, O., Clive, E., Prüm, A. y Zimmermann, R. (eds.) Principles of European Contrat Law. Part III, por la Commission on European Contract Law, Kluwer Law International, The Hague, London, Boston, 2003. La versión completa en francés: Lando, Ole y Beale, Hugh, Principles of European Contract Law. Commission pour le droit européen du contrat, version française préparée par Georges Rouhette; avec le concours de Isabelle de Lamberterie, Denis Tallon, Claude Witz, Paris, Société de Législation Comparée, 2003.

Lando, O. y Von Bar, Сh., "Communication on European Contract Law: Joint Response of the Commission on European Contract Law and the Study Group on a European Civil Code", en Europa e Diritto Privato, n. ${ }^{\circ}$ I, 2002, I33-200.

Legrand, P., "Against a European Civil Code", Modern Law Review, I997, v. $60,44-63$.

Legrand, P., "Sens et non-sens d'un Code civil européen", Revue internationale de droit comparé, 1996, v. 48, n. ${ }^{\circ} 4,779-8$ I 2.

Legrand, P., "A diabolical idea", en aA.vv., Towards a European Civil code, A. HarTKAMP et al. (ed.). Third fully revised and expanded edition, The Hague, Kluwer Law International; Nijmegen, Ars Aequi Libri, 2004, 245-2 72.

López Díaz, P. V., "Por una noción amplia de lesión en el código civil chileno: una relectura a partir del principio de equilibrio contractual y la idea de excesiva desproporción contenida en el borrador de los principios latinoamericanos de contratos", en aA.vv., Estudios de Derecho Civil X, Álvaro Vidal Olivares, Gustavo Severín Fuster, Claudia Mejías Alonzo (coords.), Santiago de Chile, Thomson Reuters, 2015, 699-723. 
Momberg Uribe, R., "Harmonization of contract law in Latin America: past and present initiatives", en Uniform Law Review, v. I9, n. ${ }^{\circ}$ 3, 20I4, 4I I-428.

Morales Moreno, A. M., "Los principios latinoamericanos de derecho de los contratos: un debate abierto sobre las grandes cuestiones jurídicas de la contratación", Anuario de Derecho civil, tomo LVviI, fasc. I, 2OI4, 22 7-254.

Nicolò, R., (voz) "Diritto civile”, en Enciclopedia del Diritto, xiI, Milano, I964, 904-92 I.

Osman, F., "Codification, unification, harmonisation du droit en Europe: un rêve en passe de devenir réalité", en Osman, F. (dir.), Vers un Code européen de la consommation? Codification, unification et harmonisation du droit des Étatsmembres de l'Union européenne: Actes et débats de Colloque: Lyon, les I 2 et I 3 décembre 1997, Bruxelles, Bruylant, I998, I I-34.

Oviedo Alban, J., "La convención sobre compraventa internacional y la modernización del derecho latinoamericano de contratos: Un tema pendiente", en Antoni Vaquer Aloy (ed. lit.), Esteve Bosch Capdevila (ed. lit.), María Paz Sánchez González (ed. lit.), El derecho común europeo de la compraventa y la modernización del derecho de contratos, Barcelona, Atelier, 20 I 5, 769-788.

Parra-Aranguren, G., "La importancia del Instituto Internacional para la Unificación del Derecho Privado (Unidroit) en la futura uniformidad jurídica del hemisferio americano", Revista de la Facultad de Ciencias Furídicas y Políticas (Caracas), n. ${ }^{\circ} 86$, I $992,33^{-73}$

Riedel, K., "The Work of the Lando-Commission from an Alternative Viewpoint", en European Review of Private Law, 2000, v. 8, n. ${ }^{\circ}$ I, 7 I-83.

Rome, F., (20I3). "Vive l'Amérique latine!", en Recueil Dalloz. n. ${ }^{41}$, 20 I 3.

Schepel, H., "Professorenrecht? Le champ du droit privé européen", en Critique Internationale, n. ${ }^{\circ}$ 26, 2005, I47-I6I.

Stadenmayer, D., "The Commission Communication on European Contract Law: What future for European Contract Law", en European Review of Private Law, 2002, v. Iо, n. ${ }^{\circ}$ 2, 249-26o.

Study Group on a European Civil Code y Research Group on the Existing EC Private Law (Acquis Group), Principles, Definition and Model Rules of 
European Private Law. Draft Common Frame of Reference (DCFR). Full Edition, III, edited by von Bar, Clive and Schulze, Munich, Sellier, 2009.

Vidal Olivares, Á. R., "Incumplimiento contractual y pretensión de cumplimiento específico en los principios latinoamericanos de derecho de contratos (PLDC)", en Antoni Vaquer Aloy, Esteve Bosch Capdevila y María Paz Sánchez González (eds. lits.), El derecho común europeo de la compraventa y la modernización del derecho de contratos, Barcelona, Atelier, 2015, 745-765.

von Bar, C.; Clive, E. y Schulte Nölke, H. (eds.), Principles, Definitions and Model Rules of European Private Law. Draft Common Frame of Reference (DCFR), Outline edition, Munich, Sellier, 2009. 